\title{
SISTEMA DE INFORMAÇÃO DA ATENÇÃO BÁSICA: POTENCIALIDADES E SUBUTILIZAÇÃO NO PROCESSO DECISÓRIO
}

Ricardo Bezerra Cavalcante', Mariana Ferreira Vaz Gontijo Bernardes² Tarcísio Laerte Gontijo $^{3}$, Eliete Albano de Azevedo Guimarães ${ }^{3}$, Valéria da Conceição Oliveira ${ }^{4}$

\begin{abstract}
RESUMO: Estudo de caso, de abordagem qualitativa, teve como objetivo analisar como profissionais e gestores têm utilizado o Sistema de Informação da Atenção Básica no processo decisório. Foram entrevistados, de janeiro a junho de 2011 e a partir de um roteiro semiestruturado, 11 profissionais que utilizam no âmbito de Secretaria Municipal de Saúde e de Superintendência Regional de Saúde, do Sistema Único de Saúde. Utilizou-se a análise de conteúdo, modalidade temática, para organização e análise dos dados. Verificou-se que o Sistema de Informação apresenta potencialidades, mas é subutilizado para o processo decisório frente à natureza do processo de trabalho e modelo da assistência, por não atender à diversidade dos modelos assistenciais e pela falta de qualificação dos profissionais. Conclui-se que há necessidade de repensar o fluxo informacional do Sistema de Informação visando o seu aproveitamento para o processo decisório local e implantando a gestão da informação como uma prática institucionalizada.

DESCRITORES: Sistemas de informação; Atenção primária à saúde; Saúde da família.

\section{THE PRIMARY CARE INFORMATION SYSTEM: POTENCIAL AND UNDER-USE IN THE DECISION-MAKING PROCESS}

\begin{abstract}
This case study, with a qualitative approach, aimed to analyze how the health professionals and managers have used the Primary Care Information System in the decision-making process. Between January and July 2011, based on a semi-structured script, interviews were held with 11 professionals who use this in the ambit of the Municipal Health Department and the Regional Health Office, of the Unified Health System. The thematic mode of content analysis was used for organization and analysis of the data. It was ascertained that the Information System has potential, but that it is under-used for the decision-making process given the nature of the work process and the model of assistance, as it does not respond to the diversity of the assistential models and because of the professionals' lack of qualification. It is concluded that it is necessary to rethink the informational flow of the Information System, with a view to its use in the local decisionmaking process and in implanting the management of information as an institutionalized practice.
\end{abstract}

DESCRIPTORS: Information systems; Primary health care; Family health.

\section{SISTEMA DE INFORMACIÓN DE LA ATENCIÓN BÁSICA: POTENCIALIDADES Y SUBUTILIZACIÓN DEL PROCESO DE DECISIÓN}

RESUMEN: Estudio de caso, de abordaje cualitativo cuyo objetivo fue analizar como profesionales y gestores vienen utilizando el Sistema de Información de la Atención Básica en el proceso de decisión. Fueron entrevistados, de enero a junio de 2011, con base en un guión semiestructurado, 11 profesionales que utilizan el sistema en el ámbito de Secretaría Municipal de Salud y de Superintendencia Regional de Salud, del Sistema Único de Salud. Fueron utilizados análisis de contenido, modalidad temática, para organización, y análisis de los datos. Se verificó que el Sistema de Información presenta potencialidades, pero es subutilizado para el proceso de decisión delante de la naturaleza del proceso de trabajo y modelo de la asistencia, por no atender a la diversidad de los modelos asistenciales y por la falta de cualificación de los profesionales. Se concluye que hay necesidad de repensar el flujo idel Sistema de Información para su aprovechamiento en el proceso de decisión local, implantando la gestión de la información como una práctica institucionalizada.

DESCRIPTORES: Sistemas de información; Atención primaria a la salud; Salud de la Familia.

${ }^{1}$ Enfermeiro. Doutor em Ciência da Informação. Bolsista CNPq de Pós-Doutorado Júnior. Professor da Universidade Federal de São João Del Rei - UFSJ.

${ }^{2}$ Enfermeira do Hospital Santa Mônica.

${ }^{3}$ Enfermeiro. Doutor em Ciências da Saúde. Professor da UFSJ.

${ }^{4}$ Enfermeira. Doutora em Enfermagem em Saúde Pública. Professora da UFSJ.

Autor correspondente:

Recebido: 28/03/2013

Ricardo Bezerra Cavalcante

Aprovado: 25/06/2013

Universidade Federal de São João Del Rei

Av. Sebastião GonçalvesCoelho, 400-35504-296-Divinópolis-MG-Brasil

E-mail: ricardocavalcanteufmg@yahoo.com.br

Cogitare Enferm. 2013 Jul/Set; 18(3):460-7 


\section{INTRODUÇÃO}

O incremento do crescimento populacional, a constante demanda por assistência à saúde e sua complexidade, desencadearam aumento exponencial de dados nos serviços de saúde. Este intenso volume de dados e informações tem gerado dificuldades para o processo gerencial e assistencial, pois os registros em saúde ainda são norteados, predominantemente, pela cultura escrita proporcionando informações precárias para direcionar as decisões gerenciais e apoiar as ações assistenciais ${ }^{(1)}$. Com vistas a instrumentalizar o processo decisório na gestão e na assistência em saúde, têm-se adotado o uso de Sistemas de Informação em Saúde (SIS) ${ }^{(1-3)}$.

Os SIS são utilizados para coletar, armazenar e disseminar dados que possam contribuir para o planejamento e, consequentemente, para o processo de tomada de decisão, seja na administração ou na assistência ao paciente $^{(4)}$. Na saúde, mediante um contexto de intensas transformações estruturais, processuais e gerenciais, a modernização dos SIS torna-se fundamental no sentido de acompanhar e oferecer respostas às novas e complexas demandas. Tais demandas decorrem das transformações organizacionais, as quais entre outros aspectos, têm intensificado o fluxo das informações e exigido um processo decisório descentralizado. E portanto, com uma participação mais efetiva dos diferentes atores sociais envolvidos nas decisões ${ }^{(5)}$.

O processo decisório é composto de uma cadeia de decisões tomadas por representantes em diferentes contextos, envolvendo um conjunto de processos que levam à formulação e à implementação de intervenções necessárias ao alcance das metas estipuladas ${ }^{(5)}$. Assim, esse envolve o estudo aprofundado do problema a partir de um levantamento de dados e informações confiáveis, necessitando de um sistema de informação que promova o suporte neste processo.

Para o monitoramento e avaliação das ações e serviços, realizados na Estratégia Saúde da Família (ESF), o Ministério da Saúde implantou em 1988, junto aos municípios e Estados, o Sistema de Informação da Atenção Básica (SIAB) ${ }^{(6)}$. Esse Sistema foi criado com o propósito de subsidiar as três esferas administrativas do Sistema Único de Saúde (SUS) com informações, visando agilizar e consolidar os dados coletados pelas equipes de saúde da família ${ }^{(7)}$. O SIAB tem o potencial para detectar desigualdades, microlocalizar problemas sanitários, avaliar intervenções, agilizar o uso da informação, produzir indicadores e, consequentemente, auxiliar o processo decisório das equipes e dos gestores ${ }^{(8)}$. Desta forma, o SIAB surge para auxiliar no planejamento e na tomada de decisão dentro da atenção básica. Entretanto, é preciso verificar se o SIAB tem cumprido com o seu papel de instrumentalizar gestores e profissionais, principalmente no processo decisório em saúde.

Alguns estudos têm demonstrado que esse Sistema apresenta problemas que se mantêm desde sua criação, tais como ineficácia na coleta e interpretação de dados; falhas sua na atualização, pois os dados da população são dinâmicos; inúmeros instrumentos de coleta e duplicidade de dados; falta de dados específicos relacionados à prevenção e promoção da saúde; as fichas de cadastro não contemplam todas as necessidades da população a ser cadastrada; os dados cadastrados e entregues às secretarias municipais e estaduais não retornam para as equipes de saúde da família, dentre outros $^{(9-10)}$. Acredita-se que estes problemas estejam influenciando a utilização do SIAB por profissionais e gestores, bem como dificultando a utilização desse Sistema no processo decisório em saúde. Assim, torna-se importante o desenvolvimento de estudos que analisem essa hipótese. Mediante o exposto, indaga-se, como o SIAB têm sido utilizado no processo decisório de gestores e profissionais de equipes de saúde da família. Assim, o objetivo desse estudo foi analisar como o SIAB tem sido utilizado no processo decisório de gestores e profissionais.

\section{MÉTODO}

Trata-se de estudo de caso de abordagem qualitativa. Os cenários compreendidos pelo estudo foram: a equipe de saúde da família mais antiga de um município de médio porte do centro oeste mineiro (nível local); a secretaria municipal de saúde do referido município (nível municipal); a e superintendência regional de saúde da macrorregião oeste (nível estadual). Estes cenários foram envolvidos devido à necessidade de compreender a utilização do SIAB seguindo o seu próprio fluxo informacional, que se inicia em uma equipe de saúde da família passando por uma secretaria municipal de saúde, até a superintendência regional.

A coleta de dados se deu por meio de entrevistas semiestruturadas no período de janeiro a junho de 2011. O roteiro de entrevista continha seis questões abertas sobre a percepção dos profissionais em relação ao sistema, e são elas: O que significa o Sistema de Informação da Atenção Básica?; A partir dos dados do SIAB você considera ser possível realizar o planejamento 
local das ações em saúde?; Você utiliza o SIAB para tomar decisões no seu cotidiano de trabalho?; Existem dificuldades para a utilização dos dados do SIAB no processo de trabalho?; Quais seriam as alterações necessárias para o aperfeiçoamento do SIAB visando a tomada de decisões?; Os profissionais recebem alguma capacitação sobre a coleta e manipulação de dados do SIAB? Qual o conteúdo desta capacitação?; e Como ela é feita?. As entrevistas foram audiogravadas e posteriormente transcritas na íntegra.

Foram incluídos 11 participantes distribuídos no nível local, municipal e estadual. No nível local foram entrevistados todos profissionais de uma equipe de saúde da família (médico, enfermeiro, técnico em enfermagem, dentista e dois agente comunitário de saúde), exceto dois agentes comunitários de saúde que não autorizaram sua participação no estudo. No nível municipal (secretaria municipal de saúde) entrevistou-se o coordenador do setor sanitário responsável por esta equipe, o diretor da atenção primária, a referência técnica de enfermagem e o auxiliar administrativo responsável pela alimentação do SIAB. No nível estadual (superintendência regional de saúde) entrevistou-se o coordenador da Atenção Primária à Saúde responsável pelo município em questão. Ressalta-se que os participantes recrutados para o estudo atuavam em seus cargos há um ano ou mais e participavam do fluxo informacional do SIAB. Os demais profissionais que não utilizavam o SIAB, não o conheciam, não participavam do fluxo informacional do SIAB, bem como estava há menos de um ano no cargos foram excluídos.

É importante ressaltar que no município envolvido no estudo há 17 equipes de saúde da família, entretanto, foi incluída no estudo apenas a primeira equipe implantada no município. Espera-se que diante de uma equipe mais antiga os fluxos e processos relacionados ao SIAB estejam mais consolidados e que os profissionais desta equipe já tenham experiência em utilizar os dados do Sistema. Realizou-se a coleta de dados no local de trabalho dos participantes. As entrevistas foram previamente agendadas e os entrevistados foram esclarecidos sobre a natureza da pesquisa, e os aspectos éticos e legais. Identificaram-se os discursos pela letra E seguida de número (1 a 11) correspondente ao entrevistado, sequencialmente.

Para organização e análise das entrevistas foi utilizado a Análise de Conteúdo, modalidade temática ${ }^{(11)}$. A escolha deste método de análise pode ser explicada pela necessidade de ultrapassar as incertezas consequentes das hipóteses e pressupostos, pela necessidade de en- riquecimento da leitura por meio da compreensão das significações e pela necessidade de desvelar as relações que se estabelecem além das falas propriamente ditas.

Este estudo foi aprovado por Comitê de Ética em Pesquisa segundo parecer n. 61/2010 e obedece aos princípios éticos da Resolução n. 196/96 do Conselho Nacional de Saúde, tendo todos entrevistados assinado o termo de livre consentimento.

\section{RESULTADOS}

A partir da análise temática, emergiram duas categorias a seguir apresentadas.

\section{Potencialidades do SIAB no processo decisório em saúde}

Foi possível identificar, a partir das falas dos entrevistados, que o SIAB disponibiliza dados que retratam a realidade dos indivíduos assistidos. Esses dados são coletados dentro do próprio domicílio, principalmente pelo Agente Comunitário de Saúde (ACS). É feita a descrição dos domicílios, com os membros da família, condições sócioeconômicas, bem como a existência ou ausência de patologias que são questões consideradas prioritárias para intervenções.

Os dados exigidos pelo o SIAB durante as visitas a gente colhe e a coleta é feita principalmente pelo agente comunitário [...] nós também realizamos as visitas, mas eles são os que ficam mais próximos [...]. Temos também os quadros que cada agente comunitário tem a situação descrita, o retrato de cada domicílio. (E1)

Você coleta os dados e entende qual a população que você tem [...]. Então, se for seguido dentro dessa normalidade, esse conhecimento da realidade te dá uma noção de planejamento. (E2)

Neste sentido, os profissionais buscam por meio das informações geradas pelo SIAB, conhecimento do que se passa com o usuário, bem como das situações que o envolvem, visando intervenções que levem à prevenção e promoção da saúde. Assim, o indivíduo em análise deve estar dentro de um quadro esperado conforme relatado:

Vou dar um exemplo: o número de gestantes. Então, vamos lá, tem tantas gestantes e tal gestante não está fazendo o acompanhamento. Por que não está fazendo? 
Isso que dá para a gente estar analisando. Aí, eu vou olhar, mas essa aqui não está fazendo o acompanhamento, por quê? Onde ela está? Ou essa criança que não está com a vacina em dia por quê? Essa daqui nasceu e não está com o aleitamento exclusivo, por quê? Então, nesse sentido o SIAB ajuda bastante a gente. (E2)

Para os profissionais, além do olhar sobre os indivíduos que o SIAB proporciona, também é possível olhar o coletivo, a comunidade onde se encontram os indivíduos. Com esse enfoque é possível realizar um diagnóstico da comunidade, de suas condições sócio-econômicas, os riscos ambientais, a estrutura da rede assistencial, a situação sanitária e epidemiológica. Nas falas dos entrevistados percebe-se que o SIAB se configura como um instrumento potencial para traçar diagnósticos que viabilizem o planejamento local.

O SIAB é um levantamento estatístico das condições de vida, de moradia da comunidade. (E8)

O SIAB pretende traçar um diagnóstico. Então, como a palavra fala, o diagnostico é para isso mesmo. Você coleta os dados, entende qual a população que você tem, planeja e articula algum plano de ação para ser feito [...]. Esse conhecimento da realidade te dá uma noção de planejamento para você traçar uma meta. (E2)

Apesar dos entrevistados apontarem as possibilidades de utilização do SIAB para a tomada de decisão, na prática, verifica-se apenas a coleta e envio de seus dados para as demais instâncias. Os dados do SIAB não são utilizados no processo de tomada de decisão local, como relatos:

Eu fico muito mais na rotina de atendimento de consulta do que avaliando o dado. A gente nem avalia, né? Não pega os dados do SIAB e discute. Preenche e manda. A gente não trabalha com os dados para mudar muito a ação não. (E8)

Esses dados do SIAB são mais para a gente fechar a produção e mandar. Você não pega o SIAB para trabalhar no seu dia-a-dia não [...]. Para melhorar tinha que dar um jeito de nós trabalharmos direto com ele. Porque, nós pegamos no SIAB somente para fechar a produção. Você coloca no SIAB o número da população, quantos diabéticos, crianças, hipertensos, gestantes, e só. Depois você não mexe mais com ele. (E9)

\section{Aspectos que podem contribuir para a subutilização do SIAB no processo decisório}

Nesta categoria são abordados alguns aspectos que podem contribuir para a subutilização do SIAB no processo decisório local. Entretanto é importante salientar que os aspectos verificados são aplicáveis apenas aos cenários e participantes deste estudo.

Um primeiro aspecto é o fato do cotidiano de trabalho dos profissionais ser focado na tarefa e produção de dados a serem enviados ao nível central. Assim, os profissionais coletam os dados do SIAB, mas não os utilizam no momento da tomada de decisão local.

A rotina da unidade é tarefista [...]. Nós fomos formados para produzir e não para sentar e achar que sentar, conversar, planejar também é trabalho [...]. Então a tarefa às vezes não faz a pessoa pensar, porque o tarefismo ele é impensado. (E2)

Outro aspecto é a ênfase dada a um modelo de assistência focada no fluxo das consultas, nos atendimentos individuais, solicitação de exames e realização de procedimento, o que reitera a lógica da assistência clínica, individual e curativa. Assim, o SIAB torna-se um sistema que armazena as informações deste modelo assistencialista, curativista, focado na doença e na medicalização como o centro da produção de informações.

O SIAB é um banco de dados dos movimentos, dos atendimentos que a gente faz aqui na unidade. Consulta de enfermagem, consulta médica, procedimentos, tais como, medir temperatura, pressão, vacina, toda a produção do que é feito aqui na unidade e é registrado. (E8)

Na reunião a gente vê que tem muito hipertensão, diabetes e a gente tenta fazer grupos, tarde comunitária, tudo voltado para isso. [...] você vai trabalhar de acordo com a realidade da população, doença da população. (E9)

O terceiro aspecto que reforça a subutilização do SIAB é a sua incapacidade de atender à diversidade (hibridismo) de modelos assistenciais presentes na atenção 
básica. Apesar do SIAB não ter sido desenvolvido para atender esse hibridismo de modelos, o sistema precisa satisfazer a diversidade informacional presente na atenção básica. Este é citado pelos entrevistados que complementam os relatos referindo a desatualização das fichas do SIAB, demonstrando, assim, não atender totalmente a demanda da ESF:

Praticamente toda a população do Brasil hoje é um modelo híbrido e o SIAB foi construído somente para a ESF. Então, para mim, ele não é um sistema da atenção básica. Pois a atenção básica hoje [...], ela é constituida por ESF e pela estratégia de atenção curativa voltada para atenção biomédica, que é dominante inclusive. (E4)

Eu acho que faz muito tempo que o SIAB foi formulado. Ele deve ser revisto, deve ser feito uma pesquisa para ver a necessidade do que deve ser acrescentado, o que tem que tirar. (E1)

Outro aspecto que pode contribuir para a subutilização do SIAB no processo decisório é a falta de um processo de capacitação sistemático e contínuo. O profissional, na maioria das vezes, é inserido na atividade sem treinamento prévio; ele se vê obrigado a aprender como manusear as fichas do SIAB no seu dia-a-dia, durante o trabalho. Relatos dos entrevistados demonstram dificuldades e dúvidas no preenchimento e uso das fichas que muitas vezes não são sanadas pelo manual técnico do SIAB. Segundo os entrevistados, à medida que surgem as dúvidas, os profissionais questionam a outro profissional da equipe, ou o auxiliar administrativo, responsável pela alimentação do Sistema. Entende-se também, pelas falas dos entrevistados, que o manual do SIAB tem abordagem pontual e fragmentada em determinados temas, como recortes dos relatos:

Não tem um curso especifico voltado ao SIAB. É mais a informação que vem passando. O treinamento específico do SIAB já foi falado várias vezes, mas não teve aqui, ao menos para mim não. (E6)

Sempre gera dúvidas em alguns itens. Tem alguns itens confusos do SIAB, que na verdade nunca foram esclarecidos, até mesmo no manual dele. No manual, você vai ler e tem itens vagos, como procedimentos coletivos. O que é isso? O que abrange? Então nunca se sabe, fica uma coisa meio vaga. (E1)
Os profissionais não têm essa capacitação de como mexer no SIAB, ele já entra e vai direto para a unidade e lá ele já tem aquela papelada toda, não só do SIAB, mas de fechamento mesmo mensal, ou outras questões. Então, não tem uma capacitação específica, a todo o momento que todo profissional que entra não é sentado com ele e passado. Mas as meninas do Sistema de Informação são sempre disponiveis, então, a qualquer dúvida, eles vêm e elas ajudam. E uma capacitação mesmo, no geral para os profissionais, não. (E5)

Alguns profissionais ainda relataram receber a capacitação somente no momento em que começam a trabalhar na atenção básica, não existindo um processo de educação permanente que aborde o SIAB ao longo dos anos:

Eles são capacitados quando eles tomam posse. [...] mas, tradicionalmente, e é aí que ele fica frágil, eu acho, é que as pessoas são capacitadas no dia que entra na SEMU$S A$ [secretaria municipal de saúde]. E se ficar 15 anos se "bobiar" tem uma capacitação somente. A gente precisa criar esse processo de educação continuada. (E3)

Nós já tivemos treinamento, mas foi bem antes, sobre como mexer, e essas coisas. Agora, trabalhar indice essas coisas ai eu já não me lembro, já tem tanto tempo, mas no último não foi feito nada nesse aspecto não. Mas teve há muito tempo, uns 8 anos atrás, bem no início mesmo. (E8)

Por fim, um último aspecto, os entrevistados relatam que não analisam os dados do SIAB por não terem uma formação acadêmica adequada para isto. Os profissionais e gestores reconhecem a necessidade de terem uma formação adequada para interpretar os dados, produzir informação para a tomada de decisões.

Eu acho que a gente não estudou para fazer isso. [...] A gente não foi tão a fundo na nossa formação e poderíamos ser capacitados sim. Porque não temos formação epidemiológica a esse nivel. E as pessoas não sabem trabalhar dados, nós não sabemos trabalhar dados e isso a gente aprende. (E2)

A gente precisa criar esse processo de educação continuada. Como eu já disse, eu não entendo muito do SIAB, mas quando eu vou tentar ler eu não consigo achar isso. (E3) 


\section{DISCUSSÃO}

A gestão do cuidado precisa ser norteada por decisões que envolvam todos os fatores relacionados ao atendimento dos usuários. Assim, o cuidado ao indivíduo, que é a retórica dos profissionais na área da saúde, precisa ser o centro de uma gestão efetiva ${ }^{(12)}$. Essa premissa, sem dúvida, desencadeia a busca por instrumentos que possam auxiliar no processo de trabalho das equipes de saúde para que possam otimizar o trabalho na gestão de serviços de saúde.

A partir da necessidade de olhar o indivíduo e o coletivo, o SIAB oferece à equipe da ESF a oportunidade de análise à medida que informa as condições sociais, econômicas, sanitárias e patológicas da população. Este panorama informacional tem a potencialidade de promover a visão sobre o coletivo de forma a orientar o processo de planejamento das intervenções a serem desempenhadas. Entretanto o processo decisório para ser efetivo precisa ir além do olhar que o sistema promove. É necessário partir para as análises de seus dados visando o processo decisório, envolvendo a gestão e a assistência local. O processo decisório constitui-se de um conjunto de processos, baseado em análises técnicas e escolhas racionais para realizar as intervenções necessárias visando atingir as metas traçadas ${ }^{(5)}$. Isto ainda precisa ser efetivado a partir da utilização do SIAB na equipe de saúde da família estudada. Outros estudos afirmam que a utilização do sistema é restrito a coleta de dados e a emissão de relatórios com pouco planejamento ou avaliação das ações desenvolvidas ${ }^{(13)}$. Isto pode prejudicar a assistência local e a utilização do SIAB por não atender ao seu objetivo primordial de instrumentalizar a gestão local.

O SIAB foi desenvolvido para auxiliar na gestão das informações produzidas pelas equipes de atenção básica em todo o território nacional. O SIAB é um sistema de informação territorializado que visa à identificação dos problemas relacionados à população, contribuindo para a avaliação e monitoramento das ações de promoção da saúde, prevenção das doenças e reabilitação $0^{(9)}$. Com seus princípios e diretrizes, o SUS, traz o ensejo pela territorialização e também a descentralização da gestão, bem como da assistência à saúde. Pretendia com este cenário um modelo ajustado na prevenção de doenças e promoção da saúde. Entretanto, o que se vê na atenção básica é um modelo de saúde híbrido, o qual coexiste esse modelo de vigilância à saúde com o modelo curativista ${ }^{(14)}$. Por um lado há a valorização da promoção da saúde e a prevenção de doenças com base no planejamento em saúde, por outro há a valorização da doença, do tratamento, da medicalização e dos exames ${ }^{(14)}$.

Em relação a ausência de um processo sistemático de capacitação relacionada ao SIAB, apontado pelos entrevistados, sabe-se que é necessário o seu desenvolvimento numa perspectiva da educação permanente. $\mathrm{O}$ processo de capacitação possibilita o aperfeiçoamento profissional, a criação do vínculo com a comunidade e o desenvolvimento do trabalho em equipe ${ }^{(13)}$. A capacitação é fundamental para que o profissional conheça o instrumento que irá utilizar, seus objetivos, finalidades e as várias possibilidades que o sistema oferece ${ }^{(9)}$. Assim, a coleta, armazenamento, análise e disseminação dos dados do SIAB poderão ocorrer de maneira adequada e disponibilizar dados fidedignos para o planejamento local das ações. Para que o SIAB atinja seu objetivo de territorialidade com a identificação dos problemas relacionados à população é necessário que a equipe da ESF e seus gestores compreendam desde o objetivo de cada dado coletado até a tomada de decisão. A compreensão de todo o sistema, e não apenas do preenchimento adequado das fichas do SIAB, pode ser alcançado com um processo de capacitação sistemático e contínuo ${ }^{(6,9)}$. O Ministério da Saúde reconhece a importância da educação e define que os municípios em conjunto com as outras duas esferas administrativas do SUS devem promover e gerenciar o processo de educação permanente ${ }^{(15)}$.

O contrário, uma capacitação fragmentada e esporádica pode comprometer todo o processo de coleta, armazenamento, análise e tomada de decisões inviabilizando as possibilidades que o sistema oferece ${ }^{(9)}$. A capacitação esporádica ainda pode gerar um menor envolvimento dos profissionais impedindo a mudança de comportamento quanto às possibilidades de análise dos dados para a tomada de decisão. Isto pode levar a utilizações pontuais dos dados e ao esvaziamento do planejamento local ${ }^{(13)}$.

Por fim, outra perspectiva que emerge neste estudo é a necessidade de que ocorra a formação de recursos humanos visando a gestão da informação em saúde. É preciso ampliar as discussões sobre informação em saúde, gestão da informação e os sistemas de informação nacionais dentro das universidades. Há uma necessidade de preparar os profissionais de saúde para utilizar a informação com vistas às ações planejadas. O Ministério da Saúde, por meio do documento Política Nacional de Informação e Informática em Saúde, determina em uma de suas diretrizes a necessidade de que futuros profissionais de saúde tenham a oportunidade, em seus currículos, de desenvolver habilidades 
de transformar dados em informações, conhecimentos e tomada de decisões ${ }^{(16)}$. Isto é algo que vai além da mera coleta e disseminação de dados, é preciso criar significados, fazer relações com a realidade da população que está sendo assistida, bem como traçar um planejamento das ações em saúde.

\section{CONSIDERAÇÕES FINAIS}

Apesar das potencialidades do SIAB em auxiliar o processo decisório, constatou-se que sua utilização está focada apenas na coleta e disseminação de dados a instâncias centralizadas. As análises dos dados visando à tomada de decisões não têm sido feitas. Assim, os dados acabam servindo aos níveis centrais e são subutilizados no planejamento local. É preciso desenvolver um processo de valorização da gestão da informação visando qualificar as decisões a serem tomadas. Um processo de reestruturação das formas de se coletar, armazenar, analisar e disseminar a informação. A materialização da gestão da informação em sua essência poderá definir as necessidades de informação, o controle dos processos de coleta, armazenamento, bem como as possibilidades de uso desta informação no planejamento da assistência local. É importante ressaltar a necessidade de um processo educacional formal sobre o tema, no contexto acadêmico e também no processo de trabalho de equipes de saúde da família. $\mathrm{O}$ aprimoramento do sistema de informação também se faz necessário adaptando-o ao modelo de saúde, às pessoas, ao processo de trabalho, aos fluxos e estruturas de poder previamente estabelecidas nos serviços de saúde. Por fim, é necessário suplantar o problema estrutural onde o excesso de atividades impede o planejamento e tomada de decisões a partir da análise crítica dos dados coletados.

\section{REFERÊNCIAS}

1. Cavalcante RB, Bernardes MFVG, Cunha SG, Santos CS. Fatores dificultadores na utilização de um Sistema de Informação em Unidades de Terapia Intensiva. R. Enferm. Cent. O. Min. [Internet] 2011;1(1) [acesso em 20 mar 2012]. Disponível: http://www.seer.ufsj.edu.br/ index.php/recom/article/view/34/79

2. Barbosa DCM, Forster AC. Sistema de Informação em Saúde: a perspectiva e a avaliação dos profissionais envolvidos na Atenção Primária à Saúde de Ribeirão Preto, São Paulo. Cad. saúde colet. [Internet] 2010;18(3) [acesso em 20 mar 2012]. Disponível: http://www.iesc.
ufrj.br/cadernos/images/csc/2010_3/artigos/CSCv18n3_ pag424-33.pdf

3. Marin HF. Sistemas de informação em saúde: considerações gerais. J. Health Inform. [Internet] 2010;02(01) [acesso em 20 mar 2012]. Disponível: http://www.jhi-sbis.saude.ws/ojs-jhi/index.php/jhi-sbis/ article/view/4/52

4. Silva AX, Cruz EA, Melo V. A importância estratégica da informação em saúde para o exercício do controle social. Ciênc. saúde colet. [Internet] 2010;12(3) [acesso em 20 mar 2012]. Disponível: http://dx.doi.org/10.1590/ S1413-81232007000300018.

5. Wendhausen A, Cardoso SM. Processo decisório e Conselhos Gestores de Saúde: aproximações teóricas. Rev. bras enferm. [Internet] 2007;5(60) [acesso em 20 mar 2012]. Disponível: http://dx.doi.org/10.1590/S003471672007000500018

6. Santos CS, Gontijo TL, Franco ECD, Cavalcante RB. Registros de atividades no Sistema de Informação da Atenção Básica. Cogitare enferm. [Internet] 2012;17(2):331-5 [acesso em 24 nov 2012]. Disponível:http://ojs.c3sl.ufpr.br/ojs2.2.4/index.php/ cogitare/article/view/23098/18547.

7. Thaines GH, Bellato R, Faria APS, Araújo LFS. Produção, fluxo e análise de dados do Sistema de Informação em Saúde: Um caso exemplar. Texto Contexto Enferm. [Internet] 2009;18(3) [acesso em 15 dez 2012]. Disponível: http://www.scielo.br/pdf/tce/ v18n3/a09v18n3.pdf (Não encontrei o DOI)

8. Ministério da Saúde (BR). A experiência brasileira em sistemas de informação em saúde/ Ministério da Saúde, Organização Pan-Americana da Saúde, Fundação Oswaldo Cruz. Brasília: Ministério da Saúde; 2009.

9. Radigonda B, Conchon MF, Carvalho WO, Nunes EFPA. Sistema de Informação da Atenção Básica e sua Utilização pela Equipe de Saúde da Família: uma revisão integrativa. Revista Espaço Saúde. [Internet] 2010;12(01) [acesso em 18 dez 2012]. Disponível: http:// www.ccs.uel.br/espacoparasaude/v12n1/sistema.pdf

10. Bittar TO, Meneguim MC, Mialhe FL, Pereira AC, Fornazari DH. O Sistema de Informação da Atenção Básica como Ferramenta da gestão em saúde. RFO. [Internet] 2009; 14(01):77-81. [acesso em $18 \mathrm{dez} 2012$ ]. Disponível: http://www.upf.br/download/editora/ revistas/rfo/14-01/77_81.pdf

11. Bardin L. Análise de conteúdo. Lisboa: Edições 70; 2011. 
12. Hennington EA. Gestão dos processos de trabalho e humanização em saúde: reflexões a partir da ergologia. Rev. Saúde Públ. [Internet] 2008;42(3) [acesso em 18 dez 2012]. Disponível: http://www.scielo.br/pdf/rsp/ v42n3/6707.pdf (Não encontrei o DOI)

13. Marcolino SJ, Scochi MJ. Informações em saúde: o uso do SIAB pelos profissionais das Equipes de Saúde da Família. Rev. Gaúcha Enferm. [Internet] 2010;31(2) [acesso em 18 dez 2012]. Disponível: http:// seer.ufrgs.br/RevistaGauchadeEnfermagem/article/ view/11939/10241

14. Limongi JE, Menezes EC, Menezes AC. Vigilância em saúde no programa de saúde da família. Hygeia. [Internet] 2009;4(7) [acesso em $18 \mathrm{dez} 2012$ ]. Disponível: http://www.seer.ufu.br/index.php/hygeia/article/ view/16916/9322

15. Ministério da Saúde (BR). Sistema de informação da atenção básica (SIAB): indicadores 2006 / Secretaria de Atenção à Saúde, Departamento de Atenção Básica. - Brasília: Ministério da Saúde; 2009.

16. Ministério da Saúde (BR). Secretaria Executiva. Departamento de Informação e Informática do SUS. Política Nacional de Informação e Informática em Saúde: Proposta Versão 2.0 (Inclui deliberações da 12a Conferência Nacional de Saúde). Brasília (DF); 2004. 